\title{
Monitoring the Depth Penetration of Dyes in Poly (Ethylene Terephthalate) Films Using a Two Layer Based Photoacoustic Model
}

\author{
L. Olenka, A. N. Medina, M. L. Baesso, A. C. Bento*, \\ Universidade Estadual de Maringá, Departamento de Física \\ Av. Colombo 5790, 87020-900, Maringá - Paraná, Brazil \\ and A. F. Rubira \\ Universidade Estadual de Maringá, Departamento de Química \\ Av. Colombo 5790, 87020-900, Maringá - Paraná, Brazil
}

Received on 27 November, 2001

\begin{abstract}
In this work we propose a photoacoustic model based on the Rosencwaig-Gersho theory to study dyed polymer samples. The model is similar to a two layer system and it is used to obtain the thermal diffusivity and the optical absorption coefficient of one side dyed polymer foils. The tested sample was the polyethylene terephthalate dyed with disperse dye, the Blue Samarom HGS. The results showed that the dye depth penetration can be accessed from the fitted data and the dyeing process can be better controlled by using the retrieved data.
\end{abstract}

\section{Introduction}

Photoacoustic (PA) is an optical technique that has been developed to study materials which can not be investigated with conventional Transmission and Reflection methods. PA is based upon heat generated by the absorbed part of an incident light onto a probe material and it was designed to solve the difficulties presented by the conventional Optical Spectroscopy, that generally can not be applied to study very weak absorbing material, and also scattering and opaque substances. The standard theory on photoacoustic effect for solids was pioneering introduced in 1976 and it is referred to as Rosencwaig and Gersho (RG) model [1, 2]. Since then, several models and methods have been developed to exploit the effect, not only for a single layer flat sample, but also for layered samples. Helander et al. [3] have presented a spectroscopic based photoacoustic theory for layered sample in which the thermal diffusion length is used for the analysis. This model was applied to a three absorbing layers of a photographic film. Also based in the RG theory, Morita [4] obtained a general expression for a periodic temperature from the samplegas boundary for multilayered polyester film, where the relationship between the phase and the films interfaces were studied. In his simulation, the layers were performed by a polyester backing in which a colored film is fixed and studied as a function of backing thickness. Tominaga et al. [5] developed another model to mea- sure thermal parameters in a two layer solid system and pointed the existence of a particular behavior for samples having different thickness. In this case, only thermal diffusivity of the thermally thick layer can be accessed, whereas if thermal thicknesses of the layers are close, then thermal diffusivity of both layers can be accessed. Mansanares et al. [6] using a two layer model, showed that thermal diffusivity and thermal conductivity can be measured by means of a effective thermal diffusivity model. They showed that effective thermal diffusivity is strongly dependent on the thermal conductivity of each individual layer, when a glass/polymer are used as the testing sample. Amato et al. [7] showed that substrate can affect the photoacoustic response in a two layer system. They proposed a generalized theory for the two layer system in which the interaction between optical and thermal parameters must be taken into account.

Generally, the thermal oscillation due to the absorption of modulated light at a point beneath the surface of a sample, contributes to the photoacoustic signal and it is ruled by the thermal diffusion length $\mu_{i}$, defined as $\mu_{i}=\left(2 \alpha_{i} / \omega\right)^{1 / 2}$, being $\omega=2 \pi f$ and $f$ is the modulation frequency of the light. Based upon this equation, one can observe that a depth profile inspection [8] can be done if the frequency is selected.

Thus, taking these photoacoustic capability we present in this paper a theoretical model that considers a single layer of a polymer that is under dyeing process 
inside a aqueous bath. Although the total thickness is unchanged for the whole system, the proposal is faced similar to a two layer system, but considers a fractional thickness being impregnated by a disperse dye. The model is applied to some films of polyethylene terephthalate (PET) where the dye are diffused from one face of the PET. The discussion will be addressed in order to have information about the changes in the physical properties and also to monitor the depth penetration.

The theoretical expression is used for fitting parameters like optical absorption and thermal diffusivity and from the results and using the signal dependence, the depth penetration of the dye is evaluated.

\section{Photoacoustic Model}

The proposed model takes into account no thermal resistance in the interface between the dyed and non-dyed layers of the polymer. The aim is to analyze the dye penetration process, using a disperse dye. This kind of dye diffuses along the film bulk without chemical bonds, with no activation energy involved. In this way we have to consider that the sample has one layer dyed and the other non-dyed, having an interface in between that will be accepted to be well defined in thickness. For instance, we consider one-dimensional geometry for the photoacoustic cell, shown in Fig. 1. The sample is a semi-infinite solid of thickness $L=\ell_{1}+\ell_{2}$, that comprises a non-dyed layer $\left(\ell_{1}\right)$ and the dyed one $\left(\ell_{2}\right)$. The modulated incident light passes through the transparent (non dyed) layer 1 and it is mainly absorbed by the dye in layer 2. The incident power at a frequency $\omega$ is given by $I(t)=I_{0} / 2\left(1+e^{i \omega t}\right)$ and according to Beer's law we have $I(x, t)=I_{0} / 2\left(1+e^{i \omega t}\right) e^{-\beta x}$ for $x \geq 0$, where $\beta$ is the optical absorption coefficient.

By considering that all absorbed light heats the sample, the density of power at a point $x$, by unity of time and unity of area, is given by

$$
S(x, t)=-\frac{d}{d x} I(x, t)=\frac{1}{2} \beta I_{0}\left(1+e^{i \omega t}\right) e^{-\beta x} .
$$

The analytical expression for the temperature at the interface sample-gas is written based on the diffusion equation for the medium. The set of coupled diffusion equation for all involved media, for the 1-D case, can be written as

$$
\frac{\partial^{2}}{\partial x^{2}} T_{j}(x, t)-\frac{1}{\alpha_{j}} \frac{\partial}{\partial t} T_{j}(x, t)+f_{j}(x, t)=0,
$$

where $f_{j}(x, t)=S(x, t) / k_{j}, j=\mathrm{g}$ (gas), b (backing), $\mathrm{s}_{1}$ (sample-layer 1$), \mathrm{s}_{2}$ (sample-layer 2 ) and $k_{j}$ is the thermal conductivity of the medium $j$.

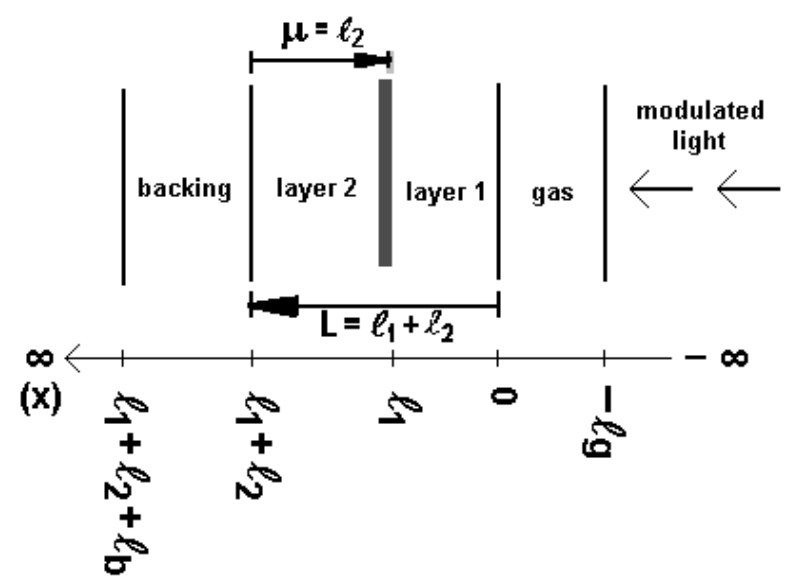

Figure 1. Geometry of the photoacoustic cell used in the 1-D dyeing penetration model.

Considering only one side of the sample being dyed we have:

$$
\begin{array}{cc}
\frac{\partial^{2}}{\partial x^{2}} T_{1}(x, t)-\frac{1}{\alpha_{1}} \frac{\partial}{\partial t} T_{1}(x, t)+\frac{\beta_{1} I_{0}}{2 k_{1}} \eta_{1} e^{-\beta_{1} x}\left(1+e^{i \omega t}\right)=0, & 0 \leq x \leq l_{1} \\
\frac{\partial^{2}}{\partial x^{2}} T_{2}(x, t)-\frac{1}{\alpha_{2}} \frac{\partial}{\partial t} T_{2}(x, t)+\frac{\beta_{2} I_{0}}{2 k_{2}} \eta_{2} e^{-\beta_{2} x}\left(1+e^{i \omega t}\right) e^{-\beta_{1} l_{1}}=0, & \quad l_{1} \leq x \leq l_{1}+l_{2} \\
\frac{\partial^{2}}{\partial x^{2}} T_{g}(x, t)-\frac{1}{\alpha_{g}} \frac{\partial}{\partial t} T_{g}(x, t)=0, & l_{g} \leq x \leq 0 \\
\frac{\partial^{2}}{\partial x^{2}} T_{b}(x, t)-\frac{1}{\alpha_{b}} \frac{\partial}{\partial t} T_{b}(x, t)=0, & l_{1}+l_{2} \leq x \leq l_{1}+l_{2}+l_{b}
\end{array}
$$

where $l_{i}$ is a thickness, $\eta_{i}$ is the light into heat conversion efficiency, $\alpha_{i}$ is a thermal diffusivity that is defined as $\alpha=\left(k / \rho c_{p}\right)$, in which $\rho$ is the mass density and $c_{p}$ is the heat capacity.

The solution of the set of above equation is proposed in the form: 


$$
\begin{gathered}
T_{g}(x, t)=\theta e^{\sigma_{g} x} e^{i \omega t} \quad(\text { gas }) \\
T_{b}(x, t)=G e^{-\sigma_{b}\left(x-l_{1}-l_{2}\right)} e^{i \omega t} \quad \text { (backing) }
\end{gathered}
$$

where the complex diffusion coefficient is given as $\sigma_{j}^{2}=$ $\left(i \omega / \alpha_{j}\right)$.

By using Eq. (7) in to Eq. (3) one has:

$$
C=\frac{\beta_{1} I_{0} \eta_{1}}{2 k_{1}\left(\beta_{1}^{2}-\sigma_{1}^{2}\right)},
$$

and, using Eq. (8) in Eq. to (4),

$$
F=e^{-\beta_{1} l_{1}} \frac{\beta_{2} I_{0} \eta_{2}}{2 k_{2}\left(\beta_{2}^{2}-\sigma_{2}^{2}\right)} .
$$

Equations (7), (8), (9) and (10) are coupled according to two boundary condition, namely:

a) Temperature continuity (layer1)

$$
T_{m}(x, t)=T_{n}(x, t)
$$

b) Heat flow continuity

$$
k_{m} \frac{d}{d x} T_{m}(x, t)=k_{n} \frac{d}{d x} T_{n}(x, t),
$$

in which $m$ and $n$ stand for adjacent media.

By applying the boundary conditions the coefficients $\theta, A, B, D$ and $E$, are determined and so, the temperature distribution in the photoacoustic cell is obtained as a function of the optical and thermal parameters of the sample, backing and gas. The temperature of interest here is that at the interface sample-gas, thus at $x=0$ one may have

$$
\theta(0)=A+B-C
$$

being

$$
\begin{aligned}
\theta(0)= & \left\{C \left[-2(r-s)(s+b) e^{l_{1}\left(\sigma_{1}-\beta_{1}\right)+2 l_{2} \sigma_{2}}-(r-1)(s-1)(s-b) e^{2 \sigma_{1} l_{1}}-\right.\right. \\
& (1+r)(s-1)(s+b) e^{2 l_{2} \sigma_{2}}+(r-1)(s+1)(s+b) e^{2 l_{1} \sigma_{1}+2 l_{2} \sigma_{2}}+(1+r)(1+s)(s-b)- \\
& \left.2(r+s)(s-b) e^{l_{1}\left(\sigma_{1}-\beta_{1}\right)}\right]-2 F\left[2 s\left(r_{2}-b\right) e^{l_{2}\left(\sigma_{2}-\beta_{2}\right)+l_{1} \sigma_{1}}+s-r_{2}\right)(b+s) e^{2 \sigma_{2} l_{2}+l_{1} \sigma_{1}}- \\
& \left.\left.\left(r_{2}+s\right)(s-b) e^{l_{1} \sigma_{1}}\right]\right\} /\left[(1+g)(s-1)(b-s) e^{2 l_{1} \sigma_{1}}-(g-1)(s-1)(b+s) e^{2 l_{2} \sigma_{2}}-\right. \\
& \left.(g-1)(s+1)(b-s)+(g+1)(s+1)(b+s) e^{2 l_{1} \sigma_{1}+2 l_{2} \sigma_{2}}\right]
\end{aligned}
$$

The interface parameters are given by:

$$
\begin{gathered}
b=\left(k_{b} a_{b} / k_{1} a_{1}\right), \quad g=\left(k_{g} a_{g} / k_{1} a_{1}\right), s= \\
\left(k_{2} a_{2} / k_{1} a_{1}\right), r=\left(\beta_{1} / \sigma_{1}\right), r_{2}=\left(k_{2} \beta_{2} / k_{1} \sigma_{1}\right)
\end{gathered}
$$

It is worthy to observe that the solution found for the two layer system can reproduce the standard equation of the RG model, if one considers $\ell_{1}$ or $\ell_{2}$ equals to zero. In the first case has a sample with a higher absorption coefficient (dyed layer), whereas in the latter, the sample is the original semi-transparent polymer (free of dyes layer).

\section{Experimental}

\section{III.1 Materials and method of prepara- tion}

Owing a good test sample we have sorted the commercially well known polyethylene terephthalate films, the so-called PET, that was impregnated in only one face. This polymer is of particular interest [9] because its processing depends on several condition like pressure, catalyst and temperature which may undergo films to have different physical properties. If chains are arranged to have a linear grouping, it is suitable to be 
applied in fabrics for bottles, toys and other objects. Otherwise, if PET chains are branched and interlaced, they may be suitable for producing laminates, bags, packages and also for coating wiring appliances.

The commercial interest for this material demands a great deal in improving its visual aspect and many situation requires the PET to be impregnated or dyed to have better aspects. Allied to this fact, the dyeing process for textile application is seen as a serious object of study for several researches [10-13].

In this work we have used commercial PET films with $100 \mu \mathrm{m}$ thick that were swollen in a bath of modifier N,N-dymethylacrylamide (N,N-DMAA), under temperature and time control at $85^{\circ} \mathrm{C}$ for $15 \mathrm{~min}-$ utes. After being washed and wiped they were dyed in a disperse blue dye solution, under temperature of $85^{\circ} \mathrm{C}$ and different times exposition of bathing. Bathing was performed at $2 \%$ dye concentration, in which several small bags of PET films were placed $(30 \times 30 \mathrm{~mm})$, being drawn from the bath at times 1, 5, 10, 15, 25, 30, 60, 180 and 360 minutes, successively. Ten samples were prepared in order to have dye penetration in only one side and so to be used in the photoacoustic depth profile analysis. All samples presented a very light blue color that is attributed to the absorption band of the disperse Samaron HGS (Dy Star) at about $600 \mathrm{~nm}$.

\section{III.2 Techniques of analysis}

A home made conventional photoacoustic cell built from an Aluminium block was used. The periodic heating of the sample was accomplished by using a 10 mW He-Ne laser (Uniphase, model 1135P) operating at $\lambda=632.8 \mathrm{~nm}$, modulated in the range from 4 to $100 \mathrm{~Hz}$ by a mechanical chopper (SRS, model 540) coupled in a lock-in amplifier (EG\&G, model 5110), the light was focused directly onto the PET sample into the photoacoustic cell. The microphone BK (4166 Brüel \& Kjäer $20 \mathrm{mV} / \mathrm{Pa}$ ) was coupled to the cell and its voltage output, as a function of modulation frequency, was measured using the lock-in amplifier. The method consists in varying the modulation frequency and collect data automatically by a computer.

As a consequence we obtain the thermal diffusion length $\mu$, which permits to access the surface structure and, therefore, the different layer of the sample can be defined. Once we measure $\alpha$ and $f$, the depth penetration is retrieved from the equation $\mu=(\alpha / \pi f)^{1 / 2}$.

\section{Results and discussion}

By using Eq. (16) which describes the temperature at the sample-gas interface, a set of theoretical curves was simulated and shown in Fig. 2. The thickness of the system dye-sample was considered as constant. The dyed sample is plotted as a percentage of the total thickness of the system varying from $0 \%$ to $100 \%$. For the simulation in this figure, we have used parameters from literature [2] for the backing and gas: $\alpha_{b}=0.97 \mathrm{~cm}^{2} / \mathrm{s}$, $\alpha_{g}=0.19 \mathrm{~cm}^{2} / \mathrm{s}, k_{b}=2.37 \mathrm{~W} / \mathrm{cmK}$ and $k_{g}=0.26$ $\mathrm{W} / \mathrm{cmK}$, also for the polymer's thermal conductivity [14] : $k_{1}=0.0022 \mathrm{~W} / \mathrm{cmK}$ e $k_{2}=0.0030 \mathrm{~W} / \mathrm{cmK}$. As we can see in Fig. 2, the set of curves present a minimum around a specifically frequency which we shall call it as "characteristic frequency $f_{c}$ ". This $f_{c}$ changes with the percentage of the dyed layer and it indicates that PA signal undergoes to a transition layer from the dyed layer into the non-dyed one.

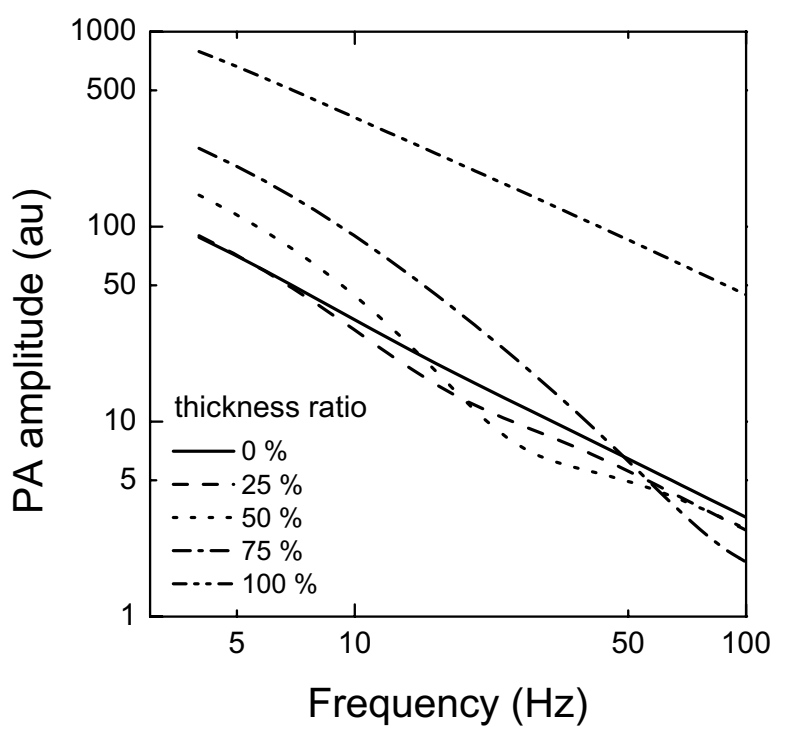

Figure 2. Theoretical simulation for different percentages of the dyed thicknesses. $\alpha_{1}=0.9 \times 10^{-3} \mathrm{~cm}^{2} / \mathrm{s}, \alpha_{2}=$ $1.1 \times 10^{-3} \mathrm{~cm}^{2} / \mathrm{s}, \beta_{1}=8 \mathrm{~cm}^{-1}, \beta_{2}=65 \mathrm{~cm}^{-1}$.

If we increase the frequency the remained signal is attributed to the surface layers. Yet, by considering that light heats the sample from the semi-transparent layer, it should be noted that the higher the $f_{c}$ the thicker is the impregnated layer. Thus, Fig. 2 shows the expected pattern for $f_{c}$ at higher frequencies for thicker dyed layer, like observed in curves simulated for $25 \%, 50 \%$ and $75 \%$. Furthermore, looking at the low frequency regime $(f<20 \mathrm{~Hz})$, the curves show higher contribution for the signal for the more absorbing sample, $100 \%$ (uppermost), compared to that with no dyes, $0 \%$ (lowest). As we increases the frequency above $f_{c}$ ( $f>50 \mathrm{~Hz}$ ), the signal is all due to an opaque layer and there is no $f_{c}$ in this case (curve for $100 \%$ ). The same observation is valid for the semi-transparent curve, 0 $\%$.

In Fig. 3 the theoretical curves are simulated for a set of $\beta$ where we note that at lower frequencies the $\beta_{2}$ contribution to the signal is more remarkable. If $\beta_{2}$ is increased from $55 \mathrm{~cm}^{-1}$ to $110 \mathrm{~cm}^{-1}$, the signal increases. This is noted by comparing curves (a) and (b) or (c) and (d). This figure also present a minimum, $f_{c}$, that is better defined for higher value of $\beta_{2}$. On the other hand, at frequencies higher than $50 \mathrm{~Hz}$, the signal for curves (a) and (b) are indistinguishable because, in this range, the signal are due to optical absorption of 
layer 1 , with $\beta_{1} \sim 8 \mathrm{~cm}^{-1}$ for both of them. The same comments is applicable for curves (c) and (d) that also have the same value of $\beta_{1} \sim 12 \mathrm{~cm}^{-1}$.

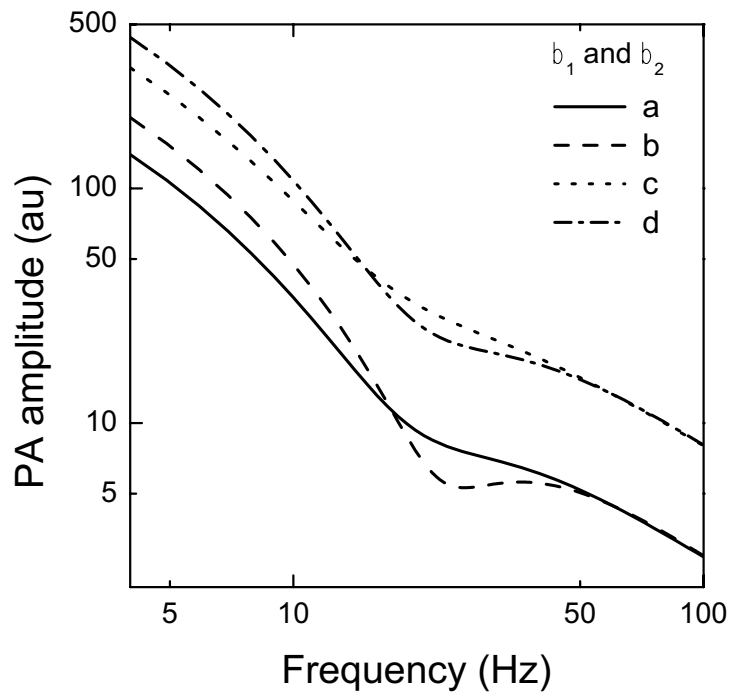

Figure 3. Theoretical simulation for different values of the optical absorption coefficient, fixing $\alpha_{1}=0.9 \times 10^{-3} \mathrm{~cm}^{2} / \mathrm{s}$, $\alpha_{2}=1.2 \times 10^{-3} \mathrm{~cm}^{2} / \mathrm{s}$; (a) $\beta_{1}=8 \mathrm{~cm}^{-1}, \beta_{2}=55 \mathrm{~cm}^{-1}$; (b) $\beta_{1}=8 \mathrm{~cm}^{-1}, \beta_{2}=110 \mathrm{~cm}^{-1}$; (c) $\beta_{1}=12 \mathrm{~cm}^{-1}, \beta_{2}=55$ $\mathrm{cm}^{-1}$ (d) $\beta_{1}=12 \mathrm{~cm}^{-1}, \beta_{2}=110 \mathrm{~cm}^{-1}$.

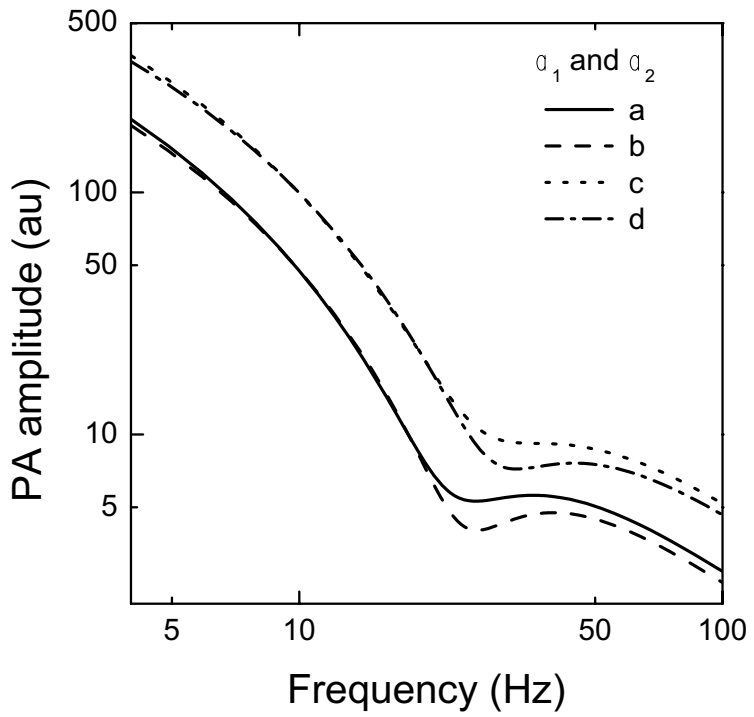

Figure 4. Theoretical simulation for different values of the thermal diffusivity, fixing $\beta_{1}=8 \mathrm{~cm}^{-1}, \beta_{2}=110$ $\mathrm{cm}^{-1}$, (a) $\alpha_{1}=0.8 \times 10^{-3} \mathrm{~cm}^{2} / \mathrm{s}, \alpha_{2}=1.2 \times 10^{-3}$ $\mathrm{cm}^{2} / \mathrm{s}$, (b) $\alpha_{1}=0.8 \times 10^{-3} \mathrm{~cm}^{2} / \mathrm{s}, \alpha_{2}=1.5 \times 10^{-3} \mathrm{~cm}^{2} / \mathrm{s}$, (c) $\alpha_{1}=1.0 \times 10^{-3} \mathrm{~cm}^{2} / \mathrm{s}, \alpha_{2}=1.2 \times 10^{-3} \mathrm{~cm}^{2} / \mathrm{s},(\mathrm{d})$ $\alpha_{1}=1.0 \times 10^{-3} \mathrm{~cm}^{2} / \mathrm{s}, \alpha_{2}=1.5 \times 10^{-3} \mathrm{~cm}^{2} / \mathrm{s}$.

The simulation of the sensitivity of this model for changes in thermal parameters is plotted in Fig. 4. Some curves are simulated for a set of values of $\alpha$ and it is noted that $f_{c}$ is shifted as $\alpha$ is changed. If $\alpha_{2}(1.2$ $\left.10^{-3} \mathrm{~cm}^{2} / \mathrm{s}\right)$ is fixed and $\alpha_{1}$ increases from $0.8 \rightarrow 1.0$ $\left(10^{-3} \mathrm{~cm}^{2} / \mathrm{s}\right), f_{c}$ also increases as shown by curves (a) and (c). The same feature is seen in curves (b) and (d). If $\alpha_{1}$ is fixed instead and $\alpha_{2}$ increases from $1.2 \rightarrow 1.5$ $\left(10^{-3} \mathrm{~cm}^{2} / \mathrm{s}\right)$ these observation are still valid if curves (a) and (b) or (c) and (d) are compared.

The dependence of the experimental signal against modulation frequency is now plotted in Fig. 5. The signal is from the Blue dye optical absorption from the HeNe laser light emitting at $632 \mathrm{~nm}$, that is very close to the absorption center of the Samaron. The set of curves in Fig. 5 show clearly that there is a $f_{c}$ higher for a thicker dyed layer of the PET. For samples exposed inside the bath for a longer time, the dye layer developed thicker, which is in agreement with the prediction of the present model, as shown in Fig. 2.

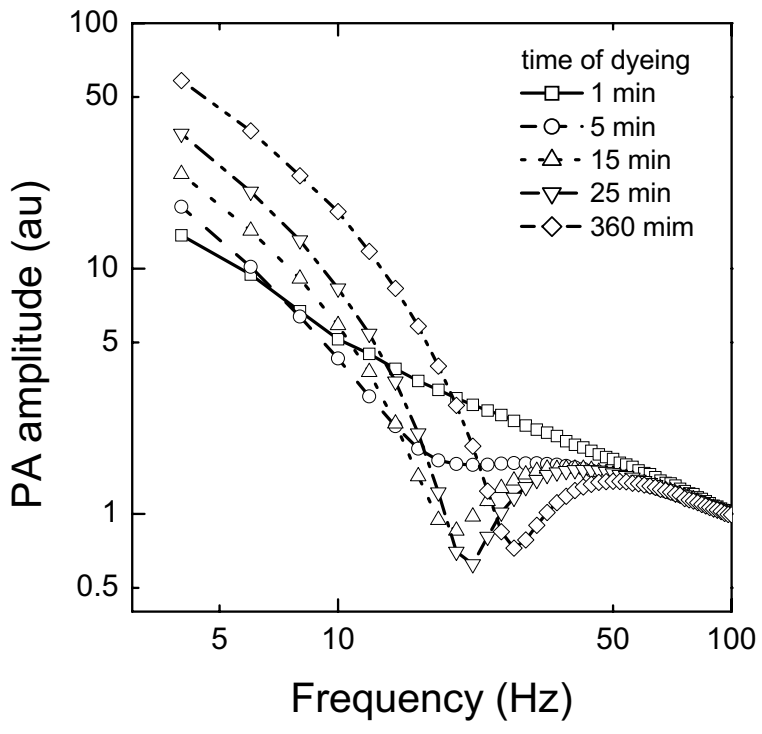

Figure 5. Experimental signal results for samples dyed at different times.

In the 1 min dyed film, there is no $f_{c}$, we may say that this time is not enough for dyeing a well defined layer of the PET film. On the other hand, for the other samples the minimum is present and becomes deeper as the optical absorption increases. This behavior was also predicted in the model and indicates that using more time in the bath means that more impregnated the PET film will be. Also, if the phases of the signal are analyzed, one can see clearly a defined distinction from sample to sample. Although we have not derived a specific expression for the phase, the experimental phases behaves like signals. Figure 6 shows PA experimental phases versus modulation frequency for a set of samples. It can be observed that the phases in this figure are similar curves that decrease in the range from 10 to $20 \mathrm{~Hz}$ showing a minimum. After the minimum they increase abruptly, reaching a maximum near $25 \mathrm{~Hz}$ and they trend to join toward a saturation above this frequency. For the sample dyed $1 \mathrm{~min}$, which practically 
has a very little dye, this tendency is almost not seen. The other observation is that the minima shift toward higher frequency as the dyeing time is increased. All curves present an increasing peak-valley distance that is correlated to the dyeing time. In this figure the solids lines are just guide to the eyes.

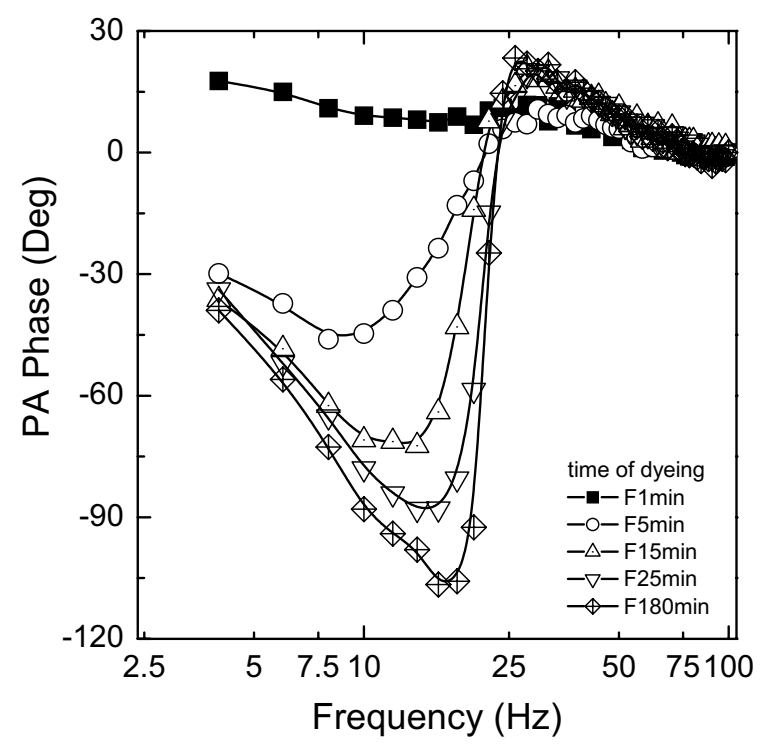

Figure 6. Experimental phase results for samples dyed at different times.

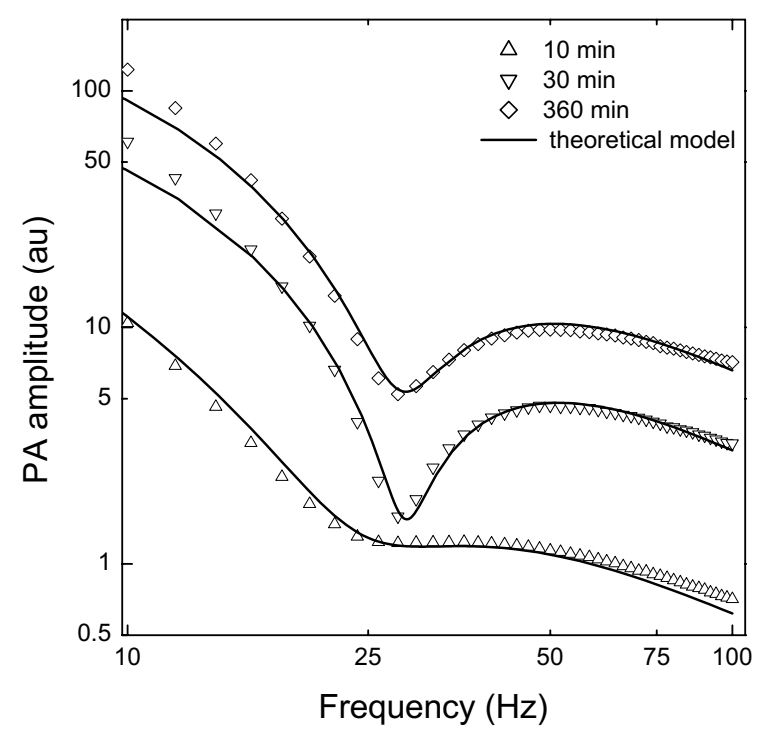

Figure 7. Fitting of the experimental data for some samples: (a) $\alpha_{1}=0.91 \times 10^{-3} \mathrm{~cm}^{2} / \mathrm{s}, \alpha_{2}=1.22 \times 10^{-3} \mathrm{~cm}^{2} / \mathrm{s}$, $\beta_{1}=7.9 \mathrm{~cm}^{-1}, \beta_{2}=105 \mathrm{~cm}^{-1}$; (b) $\alpha_{1}=0.80 \times 10^{-3} \mathrm{~cm}^{2} / \mathrm{s}$, $\alpha_{2}=1.25 \times 10^{-3} \mathrm{~cm}^{2} / \mathrm{s}, \beta_{1}=8.0 \mathrm{~cm}^{-1}, \beta_{2}=299 \mathrm{~cm}^{-1}$; (c) $\alpha_{1}=0.85 \times 10^{-3} \mathrm{~cm}^{2} / \mathrm{s}, \quad \alpha_{2}=0.99 \times 10^{-3} \mathrm{~cm}^{2} / \mathrm{s}$, $\beta_{1}=7.4 \mathrm{~cm}^{-1}, \beta_{2}=250 \mathrm{~cm}^{-1}$.

Figure 7 shows a data fitting for samples dyed for 10, 30 and $360 \mathrm{~min}$, using the expression for the tem- perature given in Eq. (16) For example, the parameters found for sample exposed $10 \mathrm{~min}$ were obtained from the best fitting for the data and the value found for the thermal diffusivity of the non-dyed layer was $0.91 \pm 0.04 \times 10^{-3} \mathrm{~cm}^{2} / \mathrm{s}$. This value is in agreement with values usually found for PET films in the literature $[15,16,17]$. For the dyed layer we found $1.22 \pm 0.06 \times 10^{-3} \mathrm{~cm}^{2} / \mathrm{s}$, showing that thermal diffusivity increased a bit with the dye. The optical absorption coefficient were also fitted for both layers and we found $7.9 \pm 0.4 \mathrm{~cm}^{-1}$ (transparent layer - PET) and $105 \pm 3$ $\mathrm{cm}^{-1}$ (dyed layer), with $\beta_{2} \approx 13 \times \beta_{1}$ in this case.

By taking the value of $f_{c}$ from some of the used samples and from $\mu=(\alpha / \pi f)^{1 / 2}$, one can calculate $\mu$ and then, evaluate the depth penetration of the dye. In Fig. 8 we show the results for the calculated dye penetration against time of dyeing. The solid line represents the fitted curve for the data using the empiric expression $\mu(t)=A[1-\exp (-t / \tau)]$. The saturation was found to be $A \simeq 62 \mu \mathrm{m}$ and the characteristic dyeing time was fitted to $\tau \simeq 25 \pm 3 \mathrm{~min}$. This result pointed that more than $50 \%$ of the sample thickness is dyed very after 25 min, showing that if sample were dyed on both face, this time guarantees a whole impregnated film.

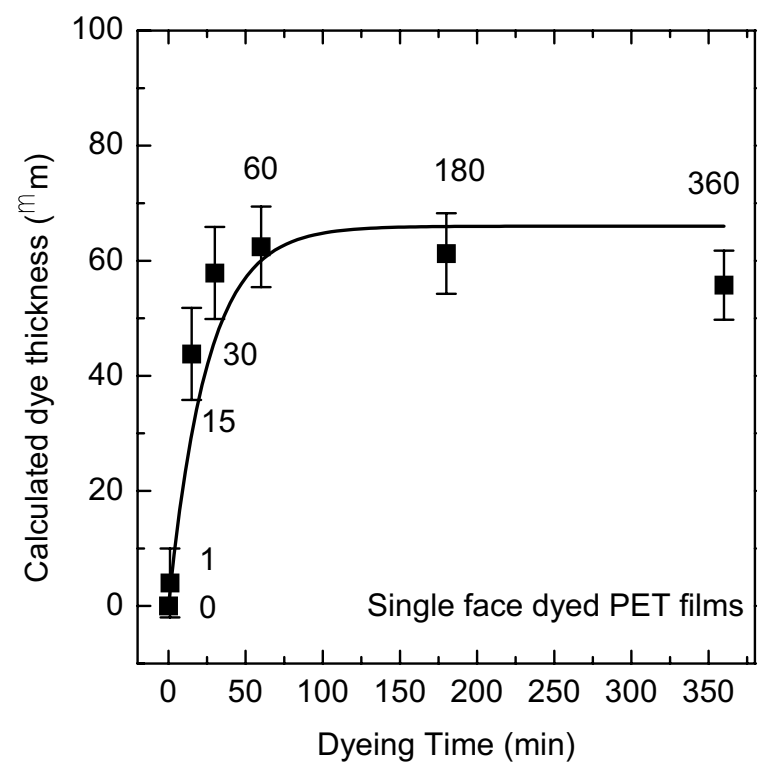

Figure 8. Kinetics for the dyeing time, $A \simeq 62 \mu \mathrm{m}$ and $\tau \simeq 25 \mathrm{~min}$.

On the other hand, the experimental results did not show the total impregnation of the film as expected in the model (see Fig. 2, curve $100 \%$ ). Despite the relatively long time of 360 min being used, we evaluated only $60 \mu \mathrm{m}$ for the impregnated layer in this case. Although we believe the time is quite enough for dyeing the whole sample, it looks that the dye is stacking in the bulk of PET film, even considering the treatment with N,N-DMAA It is expected the intumescence of the PET film in order to facilitate the dye penetration and some evidence of that already have been found [18]. 
Results for long time of dyeing is pointing that dye penetration is fast at first and slows down after some time. This effect, if it is true, might be reflecting the reducing of the opened channels in the PET bulk as the time is going. The consequence of that is very serious once this may be understood as a non homogeneous dyeing.

In our model it is assumed that dye penetration is homogeneous but the unexpected penetration for the sample dyed for 360 min suggests the existence of a gradient in the dye diffusion in the PET film bulk. This point can be seen in Fig. 5, where sample dyed during $360 \mathrm{~min}$ presented the characteristic frequency $\left(f_{c}\right)$ a little higher than the others, say $f_{c} \simeq 30 \mathrm{~Hz}$, whilst it was expected a frequency somehow near $80 \mathrm{~Hz}$ or 100 $\mathrm{Hz}$, just like simulated in Fig. 2, curve for $75 \%$ dyed sample. Besides, the minima in Fig. 5 are much more pronounced than that predicted in the model and a possible explanation for that might be in the treatment process. We have no guarantee that the treatment of the PET produced intumescence in the whole volume of the film, although the treatment is sure effective [18]. The information about the treatment depth is not available for us at this time. Under this statement one would ask if the Fick's law is not being broken. In fact, the Fick's law demonstrates that a diffusion of any substance throughout a continuous medium occurs when there exists a concentration gradient of atoms of the impurity or molecules (in our case is a disperse dye) in the solid. In equilibrium condition, this impurity should diffuse uniformly, so that a continuity law, or Fick's law rules the flux of these atoms or molecules into the solids. On the other hand, samples studied in this paper presented saturation after 30 minutes and behave like that for times up to 6 hours. We do not believe Fick's law has been broken because the dye do not diffuses until the other face of the film. It can be related to the previous treatment of the films, where the solvent makes channels to allow easy penetration of the dye that is hold in the voids of the polymer film. Thus, the main reason for the saturation might be related to the fact of films being pre-treated in only one side. We stress that this was made in order to follow the dye penetration at one side; otherwise we would not be able to evaluate the penetration.

\section{Conclusion}

The two layer similar model proposed here showed to be reliable for analyzing impregnated polymer with disperse dyes. The theoretical expression derived for the PA signal fitted data very well, showing that, at least for disperse dyes, it may be applicable to study penetration and diffusion of liquids into a transparent solid support. By performing the frequency variation from 4 to $100 \mathrm{~Hz}$ we observe a transition frequency in the PA signal between the dyed and non-dyed layer of the polymer. This characteristic frequency is different from each sample and allow us to calculate the depth penetration $\mu$, by varying modulation frequency and from fitting thermal diffusivity. The calculated penetration showed in Fig. 7, indicate that after 30 minutes in the bath the samples is practically $50 \%$ dyed. This is due the fact that the samples are been dyed in only one face. Dyeing both sides this time may be sufficient to dye completely a $100 \mu \mathrm{m}$ polymer film. Finally, the results presented here point to a saturation in the impregnating process after swelling times about 30 minutes.

\section{Acknowledgments}

L. O. gratefully acknowledges to Capes and also to $\mathrm{CNPq}$ for the partial support in this work.

\section{References}

[1] A. Rosencwaig, A. Gersho, J. App. Phys. 47(1), 64 (1976).

[2] A. Rosencwaig and A. Guersho, Photoacoustic e photoacoustic spectroscopy, John Wiley \& Sons, New York, 1980.

[3] P. Helander, I. Lundström, and D. McQueen, J. Appl. Phys. 52(3), 1146 (1981).

[4] M. Morita, Japn. J. Appl. Phys. 20(5), 825 (1981).

[5] T. Tominaga, K. Ito, Japn. J. Appl. Phys. 27(12), 2392 (1988).

[6] A. M. Mansanares, A. C. Bento, H. Vargas, N. F. Leite, and L. C. M. Miranda, Phys. Rev. B 42(7), 4477 (1990).

[7] G. Amato, G. Benedetto, L. Boarino, M. Maringelli, and R. Spagnolo, Appl. Phys. A 52, 280 (1981).

[8] M. G. de Oliveira, O. Pessoa Jr., H. Vargas, and F. Galembeck, J. Appl. Polym. Sci.35, 1791 (1988).

[9] H. F. Mark, N. G. Gaylord, and N. M. Bilakes, (executive editor consultant); Encyclopedia of Polymer Science and Technology, vol.11, (Interscience Publishers, 1969).

[10] A. Bendak, S. El-Marsafi, Annali de Chimica, 82, 603 (1992).

[11] H. L. Needles, C. Walker, and Q. Xie, Polymer 31, 336 (1990).

[12] F. J. C. Fité, Textile Res.J. 65 (6), 362 (1995).

[13] A. N. Saligram, S. R. Shukla, and M. Mathur, JSDC, 109, 263 (1993).

[14] L. Olenka, É. N. da Silva , W. L. F. dos Santos, E. C. Muniz, A. F. Rubira, A. N. Medina, L. P. Cardoso, L. C. M. Miranda, M. L. Baesso, and A. C. Bento, J. Phys. D: Appl. Phys. 34, 2248 (2001).

[15] P. Korpiun, B. Merté, G. Fritsch, R. Tilgner, and E. Lüscher, Colloid \& Polymer Sci. 261(4), 312 (1983).

[16] M. J. Adams, G. F. Kirkbrightm, Analyst, 102, 678 (1977).

[17] A. Lachaine, P. Poulet, Appl. Phys. Lett. 45(9), 953 (1984).

[18] W. L. F. dos Santos, M. F. Porto, E. C.Muniz, L. Olenka, M. L. Baesso, A. C. Bento, and A. F. Rubira, J. Appl. Polym. Sci. 77, 289 (2000). 